\title{
MODELO DE PREVISÃO DE VENDAS EM JOGOS DE EMPRESAS: POTENCIALIZANDO A PRÁTICA DOS GESTORES
}

\section{SALES FORECAST MODEL IN BUSINESS GAMES: LEVERAGING MANAGERIAL PRACTICE}

\author{
Recebido em 20.04.2015. Aprovado em 23.05.2016 \\ Avaliado pelo sistema double blind review \\ DOI: http://dx.doi.org/10.12712/rpca.v10i2.578
}

\author{
Sheila Serafim da Silva \\ sheila_serafim@yahoo.com.br \\ Universidade Federal Fluminense (UFF), Volta Redonda/RJ, BRASIL
}

\section{Murilo Alvarenga Oliveira}

malvarenga@id.uff.br

Universidade Federal Fluminense (UFF), Volta Redonda/RJ, BRASIL

\author{
Ilton Curty Leal Junior \\ iltoncurty@gmail.com \\ Universidade Federal Fluminense (UFF), Volta Redonda/RJ, BRASIL
}

\begin{abstract}
Resumo
A previsão de demanda desempenha um papel importante nas organizações, pois auxilia o gestor no processo de tomada de decisão. Em geral, as empresas não dispõem de recursos suficientes para alterar de forma substancial a oferta no curto prazo e atender a oscilação da demanda. Diante disso, este estudo teve como objetivo desenvolver um modelo de previsão de vendas para uso em um jogo de empresas. A pesquisa abordou a técnico jogo de empresas como instrumento de pesquisa. Foi utilizada uma abordagem quantitativa com uso da análise de regressão linear múltipla. $\mathrm{O}$ modelo foi testado em um ambiente laboratorial no período de um ano simulado. Foram analisados e discutidos os erros na previsão de vendas com e sem o uso do modelo. Os principais resultados apontaram que o modelo contribuiu para melhorias no desempenho da empresa analisada sob a perspectiva operacional e financeira, sendo o erro considerado aceitável. O modelo foi capaz de prever a demanda obtendo-se um erro inferior às empresas que não usaram o modelo. Pôde-se verificar benefício decorrente do uso de um método estatístico para antecipar e prever demanda, uma vez que o modelo de previsão agregou valor à empresa, destacando-se a possibilidade de usar tais técnicas ou outras para antecipar a demanda futura. Entre as principais contribuições do estudo, foi destacado o uso de ambientes de simulação para teste de modelos e teorias na área de produção e operações para a produção de novos conhecimentos.
\end{abstract}

Palavras-chave: Previsão de Vendas. Jogo de Empresas. Laboratório de Gestão.

\begin{abstract}
Demand forecasting has an important role in organizations and assists the manager in the decision making process. This study aims to develop a demand forecast model and preliminary test on a business games. Thus, this study aimed to develop a sales forecast model for use in a business game. The study addressed the game companies as a research tool. A quantitative approach using multiple linear regression analysis was used. The model was tested in an environment within one simulated year. Errors were discussed in the sales forecast with and without the use of the model. The main results showed that the model has contributed to improvements in company performance analyzed under the operational and financial perspective, and the error considered acceptable. The model was able to predict the demand resulting in an error lower than companies that didn't use the model. It was verified benefit from the use of a statistical method to anticipate and predict demand, since the forecast model added value to the company, highlighting the possibility of using such techniques or other to anticipate future demand. The main contribution of the study was the use of simulated environments to test concepts and theories in the area of production and operations for the production of new knowledge.
\end{abstract}

Keywords: Demand Forecasting. Business Games; Management Laboratory. 


\section{Introdução}

A previsão de vendas desempenha um papel importante no processo de tomada de decisão nas organizações. Em geral, as empresas não dispõem de recursos suficientes para alterar de forma substancial a oferta no curto prazo e atender as oscilações da demanda. Apesar de amplamente estudada, a previsão de vendas é um fenômeno complexo que envolve uma série de variáveis endógenas e exógenas às organizações.

Erros na previsão de vendas dificulta o planejamento nas diversas áreas funcionais, o que resulta em perdas financeiras e impacta na competitividade da empresa frente os concorrentes (GERBER et. al., 2013). A previsão de vendas busca o alinhamento das necessidades do mercado à capacidade de resposta da empresa. Todavia, isto representa um problema devido à falta de informações e forte influência das variáveis exógenas à organização (MELO; ALCÂNTARA, 2011).

A ausência de um sistema de previsão de vendas conduz o gestor à tomada de decisão por intuição, o que pode ser prejudicial e resultar em ineficiência mercadológica. Por isso, diversos modelos computadorizados com a abordagem de métodos quantitativos e qualitativos têm sido desenvolvidos para o cálculo da demanda.

Uma pesquisa recente na literatura demonstrou a existência de estudos que tratam de modelos de cálculo de demanda em jogo de empresas. Um estudo realizado por Gold e Pray (1982) comparou as funções do modelo de cálculo de demanda de oito jogos de empresas e discutiu de forma teórica e empírica com base na microeconomia os aspectos que devem ser incorporados nesse tipo de modelo. Outros estudos propuseram novos modelos de cálculo da demanda em simulações, sendo os principais avanços realizados por Gold e Pray (1984) e Thavikulwat (1988).

Todavia, há poucos estudos que investigam o desenvolvimento de modelos de cálculos de demanda do ponto de vista do participante de um jogo de empresas (HONAISER; SAUAIA, 2008). Um estudo recente realizado por Honaiser e Sauaia (2008) discutiu os modelos de séries temporais (extrapolação e suavização exponencial), modelos causais explicativos (correlação, regressão e modelos econométricos) e avaliação subjetiva (entrevista com executivos) e propôs o desenvolvimento de um modelo de previsão de demanda do ponto de vista de um participante do jogo de empresas.

Cabe destacar que um jogo de empresas consiste em uma experiência vivencial em que o participante se torna gestor de uma empresa fictícia, toma decisões acerca de um produto e compete com outras empresas (SAUAIA, 1995).

A previsão da demanda é uma tarefa fundamental que antecede a formulação da estratégia das empresas. A ausência de uma previsão de demanda ou uma gestão da demanda ineficiente pode resultar em excesso ou falta de demanda. Para reduzir esse risco, buscou-se responder a dois questionamentos: (a) qual o modelo mais adequado de previsão de vendas pode apoiar os gestores participantes de um jogo de empresas? (b) o modelo contribui para melhorias no desempenho da empresa analisada?

Diante disso, este estudo teve como objetivo desenvolver um modelo de previsão de vendas adequado ao jogo de empresas e comparar a previsão com o volume de vendas real antes e após o uso do modelo de previsão de vendas. Para a comparação com o uso do modelo, a simulação foi reprocessada considerando as novas decisões tomadas a partir do modelo de previsão desenvolvido e todas as demais decisões permaneceram inalteradas.

A partir dessa introdução, este estudo está estruturado como se segue: a segunda seção apresenta a revisão de literatura acerca das técnicas de previsão de vendas e uso dos jogos de empresas para produção de pesquisa; a terceira seção apresenta os procedimentos metodológicos seguidos na elaboração deste estudo; a quarta seção descreve o ambiente simulado no qual a pesquisa foi realizada; a quinta seção descreve o modelo de previsão de vendas; a sexta seção apresenta a análise descritiva dos resultados; a seção 8 discute os resultados; a seção 9 apresenta as considerações finais.

\section{Técnicas de Previsão de Vendas}

A previsão de vendas consiste na antecipação da demanda futura por meio de um plano de vendas exequível no longo prazo. Tal plano deve estar alinhado com o plano-mestre de produção e servir de base para as demais decisões da empresa. Todavia, 
exige o uso de técnicas que sejam capazes de prever e antecipar a demanda futura.

De acordo com Lin (2000), as técnicas de previsão de vendas podem ser divididas em qualitativas e quantitativas. As técnicas qualitativas são avaliações subjetivas por meio do julgamento de especialistas e fazem uso do método Delphi, pesquisa de mercado e pesquisa de opinião. Por outro lado, as técnicas quantitativas se baseiam na construção de modelos matemáticos a partir de informações históricas e podem ser divididas em séries temporais e causais ou explicativas (SAUAIA; HONAISER, 2008).

De acordo com Proto e Mesquita (2003), as séries temporais consistem em métodos de projeção, os quais assumem que a demanda futura será uma reprodução do passado, como exemplo, a média móvel, suavização exponencial e autocorrelação. Enquanto, as séries causais consistem em modelos de regressão, os quais são baseados na premissa de que a mesma relação de dependência entre as variáveis explicativas (independentes) e a demanda (variável dependente) irá permanecer no futuro, como exemplo, análise de regressão simples e múltipla e os modelos econométricos.

De acordo com Honaiser e Sauaia (2008), as técnicas de análise de regressão tem um papel importante quando se trata de previsão de vendas em uma simulação de negócios, isso se deve ao fato de que os modelos analíticos de previsão de vendas utilizados em simulações de negócios são dependentes de diversas variáveis econômicas.

Contudo, a previsão baseada apenas em dados históricos ou na opinião de especialistas pode não alcançar o nível de precisão desejado. A demanda depende de uma série de fatores e estes podem não ser levados em consideração por uma única técnica. Os autores Clemen (1989) e Goodwin (2002) discutem a combinação de técnicas quantitativas e qualitativas para melhorar o modelo de previsão. Após a construção do modelo objetivo, este deve passar por um ajuste de julgamento de especialistas.

Um estudo realizado por Werner e Ribeiro (2006) discute uma série de combinações de técnicas para ajustes no modelo. Entre eles, o estudo de Webby e O’Connor (1996) que propõe uma análise subjetiva dos dados históricos em paralelo à construção do modelo objetivo. Desta forma, é possível construir um modelo de previsão baseado em informações contextuais. Todavia, deve-se avaliar a eficácia dos ajustes pela opinião do especialista e como eles podem ser melhorados (SYNTETOS et. al., 2009).

Os métodos de projeção fazem uso da extrapolação para realizar as previsões futuras com base em dados históricos, mas sem tentar identificar os aspectos que o influenciam. Contudo, os modelos de regressão buscam identificar quais as variáveis estão correlacionadas à demanda e o quanto estas variáveis a influenciam. Por isso, este estudo utilizará a técnica de análise de regressão para prever a venda futura da empresa em um jogo de empresas.

Cabe destacar a diferença entre previsão de demanda e previsão de venda. A primeira compreende a procura por um bem ou serviço, enquanto a segunda consiste na estimativa do bem ou serviço que a empresa espera atender no mercado.

\section{Jogos de Empresas como Instrumento de Pesquisa}

O jogo de empresas consiste em uma experiência vivencial a qual o participante vivencia situações de um ambiente empresarial real por meio de um processo de tomada de decisão (SAUAIA, 1990). O jogo de empresas pode ser definido como um exercício que recria ambientes experimentais no qual as mudanças no aprendizado e no comportamento gerencial podem ser observadas e estudadas (KEYS; WOLFE, 1990).

Por se tratar de um ambiente simplificado com situações verossímeis da realidade que induz à respostas semelhantes ao ambiente real pelos participantes (KEYS; WOLFE, 1990), o jogo de empresas foi criado para fins educacionais, tendo como intuito a aproximação da teoria à prática da profissão de administração. Um estudo realizado por Ben-Zvi (2010) discutiu o uso de jogo de empresas em um ambiente educativo para a aprendizagem e implementação de sistemas de informação de gestão eficazes.

É possível testar teorias e modelos do mundo empresarial com o apoio do jogo de empresas, sendo proposto por Keys e Wolfe (1990) o uso do jogo de 
empresas como laboratórios de pesquisa. Todavia, o uso do método orientado para a pesquisa se limitava à provisão de dados para pesquisas realizadas por docentes (OLIVEIRA, et. al. 2011).

Ao observar um ambiente propício para realização de pesquisas pelos próprios educandos, Sauaia (2008) propôs o uso do jogo de empresas dentro de laboratórios de gestão. A proposta de laboratórios de gestão foi desenvolvida por Sauaia (2008), com a aplicação de jogos de empresas e pesquisa na área, observando que em outras áreas de estudo os educandos são participantes ativos no ensaio de problemas e soluções.

Nesse estudo, o jogo de empresas foi utilizado no contexto de um laboratório de gestão como ambiente de aprendizagem, no qual os participantes tiveram a oportunidade de vivenciar uma experiência próxima da realidade ao se deparar com problemas organizacionais complexos que ocorrem no cotidiano das organizações e, em seguida, solucionar um problema empresarial por meio de investigação e produção científica.

\section{Procedimentos Metodológicos}

Esse estudo trata-se de uma pesquisa aplicada com uso de procedimento bibliográfico e pesquisa documental. Os dados foram coletados em relatórios de quatro empresas participantes de um jogo de empresas. A técnica adotada neste estudo é quantitativa e está baseada em modelos causais, com uso da análise de regressão linear múltipla.

O uso da análise de regressão justifica-se por meio de dois fatores: primeiro, o simulador utilizado possui um número considerável de variáveis que podem influenciar na demanda. Segundo, pelo fato de ter sido amplamente utilizada em outros estudos, inclusive em ambientes laboratoriais com uso de jogo de empresas (HONAISER; SAUAIA, 2008). Técnicas qualitativas não foram utilizadas pela ausência de especialistas em jogo de empresas que pudessem julgar o comportamento da demanda com base nos dados passados. Entre as técnicas quantitativas, a de série temporal não foi considerada devido à dependência da demanda de outras variáveis além do tempo.

A análise de regressão busca identificar quais as variáveis estão correlacionadas à demanda e a influência de cada variável. Para uso do modelo de regressão linear, deve-se utilizar a Função 1.

$Y=b_{0}+b_{1} X_{1}+b_{2} X_{2}+\cdots+b_{m} X_{m}+e$
$Y=b_{0}+b_{1} X_{1}+b_{2} X_{2}+\cdots+b_{m} X_{m}+e$

Onde:

Y corresponde a variável dependente (demanda)

$X_{1}, X_{2}, \ldots, X_{m} X_{1}, X_{2}, \ldots, X_{m}$

correspondem as variáveis independentes que influenciam a demanda $e$ corresponde ao erro aleatório.

O estudo de Porter et. al. (1991) sugere que a previsão de vendas com uso de técnicas de regressão deve seguir cinco etapas: (a) seleção das variáveis que influenciam a venda; (b) coleta de dados; (c) seleção da função; (d) verificação da exatidão do modelo por meio da determinação do $\mathrm{R}^{2}$; (e) obtenção do valor projetado para o futuro algebricamente. Tais procedimentos foram adotados neste estudo.

De acordo com Proto e Mesquita (2003), a seleção das variáveis que irão compor o modelo é uma tarefa complexa em análise de regressão. De acordo com os autores, não se deve incluir no modelo as variáveis que não tem forte relação de causa e efeito com a venda e ao mesmo tempo deixar de incluir as variáveis que explicam parte desta relação. Neste ponto, devese observar a multicolinearidade entre as variáveis independentes. Uma síntese do modelo pode ser observada na Figura 1:

Figura 1. Modelo de Previsão de Vendas

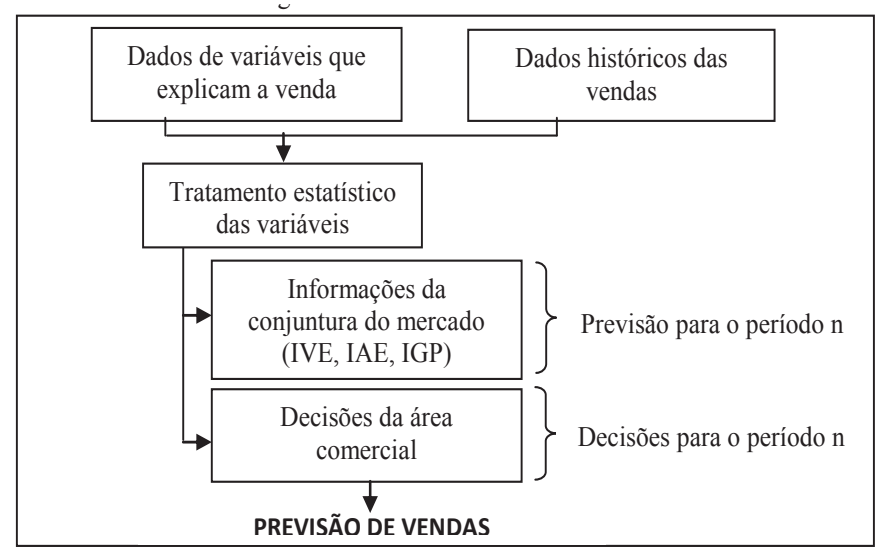

Fonte: Elaboração própria. 
Após a determinação das variáveis independentes, os dados foram coletados. Em seguida, foi aplicada a análise de regressão que determinou os coeficientes $\mathrm{b}_{1}$ 's de cada variável independente. A partir de então, estabeleceu-se a função matemática do modelo. $\mathrm{O} \mathrm{R}^{2}$ foi determinado com o intuito de conhecer a exatidão do modelo.

Após esta etapa, comparou-se a variação da previsão de vendas com o volume de vendas real sem e com o uso do modelo de previsão de venda. Nesta etapa, buscou-se verificar como o modelo desenvolvido se ajusta aos dados históricos. Por meio de uma resimulação, foram comparados os erros de previsão da venda e o impacto no desempenho da empresa analisada.

\section{Descrição do Ambiente de Simulação}

Os dados para elaboração deste estudo foram coletados em uma disciplina denominada Laboratório de Gestão Simulada em um curso de Administração de uma Instituição de Ensino Superior no Estado do Rio de Janeiro. O estudo foi realizado com base na proposta do Laboratório de Gestão que se apoia no tripé conceitual proposto por Sauaia (2008): simulador organizacional, jogo de empresas e pesquisa aplicada.

Foi utilizado o ambiente "Grego Mix" do software Shadow Manager. O software é um conjunto de simuladores em que diversos ambientes podem ser preparados de acordo com a necessidade do docente. Para simplificar, neste estudo o ambiente GregoMix será denominado "simulador".

Um simuladordenegócios trata-se de uma simplificação da realidade empresarial em que certo número de variáveis do ambiente real é envolvido (ROSAS, 2009). Em geral, são ferramentas computacionais utilizadas para propiciar a tomada de decisão e para fornecer relatórios aos participantes (SILVA; OLIVEIRA; MOTTA, 2013).

Os participantes assumem a gestão de uma empresa fictícia e se envolvem em um processo de tomada de decisão ao longo de $\mathrm{n}$ períodos. A atividade inicia com a descrição de uma situação inicial e com a apresentação de um conjunto de parâmetros:
- Visão geral do simulador e cenário econômico;

- Características gerais das empresas e dos produtos;

- Situação inicial das empresas quanto à estrutura financeira, capacidade de produção, recursos humanos, investimentos comerciais, etc.

Após o conhecimento dos parâmetros e da situação inicial, os gestores das empresas fictícias tomam um conjunto de decisões. Tais decisões (dados de entrada) ao serem processadas fornecem um conjunto de relatórios (dados de saída) que apontam o desempenho das empresas em termos comercial, operacional e financeiro. A cada rodada do jogo, as decisões são tomadas para um período trimestral com base na previsão do cenário econômico indicada nos relatórios, nas decisões e resultados públicos dos concorrentes (preço e volume de vendas) e nos resultados individuais de cada empresa.

A seguir serão descritos alguns parâmetros do Simulador Grego Mix (SGM):

O ambiente simula a relação entre empresas pertencentes à mesma cadeia de suprimentos, conforme a Figura 2. Os participantes são divididos entre empresas industriais e intermediárias (atacadistas).

Figura 2 . Cadeia de Suprimentos do Jogo

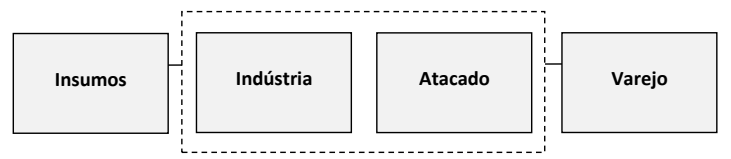

Fonte: Elaboração própria.

O cenário econômico e político simulado é estável. Para orientar os participantes, a cada período é fornecida uma previsão de três índices econômicos: Índice de Atividade Econômica (IAE), Índice de Variação Estacional (IVE) e Índice Geral de Preços (IGP). 
Demais parâmetros fornecidos são as taxas de juros sobre empréstimo, cheque especial, remuneração da conta corrente e o imposto de renda.

As empresas são oligopólio e comercializam até três tipos de produtos do setor de eletrônicos. Os produtos (alfa, beta e ômega) possuem características distintas em termos de ciclo de vida, demanda e faixa de preço. Os fornecedores de insumo para a indústria possuem condições de pagamento e preço pré-definidas.

A condição inicial em termos de capacidade de produção, capital social, estoque e despesas fixas são fornecidas às indústrias, além das regras de produção, contratação e demissão de operários. Da mesma forma, é fornecido aos atacadistas o cenário inicial com o capital social disponível, número de vendedores, despesas fixas e produtos em estoque.

As indústrias tomam decisões quanto ao tipo de produto a ser produzido, em que quantidade, prazo de recebimento, compra e venda de maquinário, contratação e demissão de operários, investimentos em benefícios e treinamento e distribuição de dividendos. Os atacadistas tomam decisões quanto ao tipo de produto a ser comercializado, preço ao varejo, prazo de recebimento, investimentos em propaganda e no ponto de venda (comercial), distribuição de dividendos, número de vendedores e salários (aumento do salário e comissão).

As transações entre indústrias e atacadistas são realizadas entre si. Em cada período, as decisões tomadas pelas empresas são processadas pelo simulador que fornece alguns relatórios gerenciais: Demonstração do Resultado do Exercício (DRE), Balanço Patrimonial, Análise do Custo e Relatório Público Geral (comum a todas as empresas).

O desempenho das equipes é medido pela Taxa Interna de Retorno (TIR). A TIR é calculada por ramo de atividade, sendo o desempenho dos atacadistas comparado com os demais atacadistas e as indústrias da mesma forma. Para fins didáticos, a taxa é convertida em uma nota e apresentada aos alunos.

\section{Descrição do Modelo de Previsão de Vendas}

Para aplicação do método fez-se necessário o cumprimento das cinco etapas propostas por Porter et. al. (1991), os quais serão detalhados adiante.

Foi realizada uma busca pelas variáveis presentes no simulador com potencial de influencia na venda. Foram levados em consideração todos os fatores quantificáveis presentes no jogo de empresas. As variáveis identificadas podem ser observadas no Quadro 1.

Quadro 1.Variáveis independentes com potencial para compor o modelo

\begin{tabular}{|c|c|c|c|}
\hline \multicolumn{2}{|r|}{ Variável } & Descrição & Unidade \\
\hline $\mathrm{X}_{1}$ & Preço & $\begin{array}{l}\text { Preço atribuído ao } \\
\text { produto } \alpha, \beta \text { e } \Omega \text {. }\end{array}$ & $\mathrm{R} \$$ \\
\hline $\mathrm{X}_{2}$ & Prazo & $\begin{array}{l}\text { Prazo de } \\
\text { recebimento dos } \\
\text { clientes para o } \\
\text { produto } \alpha, \beta \text { e } \Omega \text {. }\end{array}$ & Dias \\
\hline $\mathrm{X}_{3}$ & $\begin{array}{l}\text { Orçamento } \\
\text { promocional }\end{array}$ & $\begin{array}{l}\text { Investimento } \\
\text { destinado à } \\
\text { propaganda para o } \\
\text { produto } \alpha, \beta \text { e } \Omega \text {. }\end{array}$ & $\mathrm{R} \$$ \\
\hline $\mathrm{X}_{4}$ & $\begin{array}{l}\text { Orçamento } \\
\text { comercial }\end{array}$ & $\begin{array}{l}\text { Investimento } \\
\text { destinado à } \\
\text { promoção no } \\
\text { ponto de venda do } \\
\text { produto } \alpha, \beta \text { e } \Omega \text {. }\end{array}$ & $\mathrm{R} \$$ \\
\hline $\mathrm{X}_{5}$ & Comissão & $\begin{array}{l}\text { Comissão } \\
\text { atribuída ao } \\
\text { vendedor } \\
\text { com base no } \\
\text { faturamento para } \\
\text { o produto } \alpha, \beta \text { e } \\
\Omega \text {. }\end{array}$ & $\%$ \\
\hline $\mathrm{X}_{6}$ & $\begin{array}{l}\text { Aumento } \\
\text { salarial }\end{array}$ & $\begin{array}{l}\text { Aumento } \\
\text { salarial dado aos } \\
\text { vendedores. }\end{array}$ & $\%$ \\
\hline $\mathrm{X}_{7}$ & $\begin{array}{l}\text { Número de } \\
\text { vendedores }\end{array}$ & $\begin{array}{l}\text { Número de } \\
\text { vendedores } \\
\text { alocados para a } \\
\text { venda do produto } \\
\alpha, \beta \text { e } \Omega \text {. }\end{array}$ & Qtde. \\
\hline $\mathrm{X}_{8}$ & $\begin{array}{l}\text { Índice Geral } \\
\text { de Preços }\end{array}$ & $\begin{array}{l}\text { Mede a evolução } \\
\text { dos preços na } \\
\text { economia. }\end{array}$ & Adimensional \\
\hline $\mathrm{X}_{9}$ & $\begin{array}{l}\text { Índice de } \\
\text { Atividade } \\
\text { Econômica }\end{array}$ & $\begin{array}{l}\text { Mede a atividade } \\
\text { econômica do } \\
\text { país. }\end{array}$ & Adimensional \\
\hline $\mathrm{X}_{10}$ & \begin{tabular}{|l} 
Índice de \\
Variação \\
Estacional
\end{tabular} & $\begin{array}{l}\text { Mede a } \\
\text { sazonalidade } \\
\text { do produto no } \\
\text { período n. }\end{array}$ & Adimensional \\
\hline
\end{tabular}




\begin{tabular}{|c|l|l|c|}
\hline $\mathrm{Y}$ & $\begin{array}{l}\text { Volume de } \\
\text { Vendas }\end{array}$ & $\begin{array}{l}\text { Quantidade } \\
\text { vendida em } \\
\text { unidades do } \\
\text { produto } \alpha, \beta \text { e } \Omega .\end{array}$ & Qtde. \\
\hline
\end{tabular}

Fonte: Elaboração própria.

A partir de então, foi realizado um teste de multicolinearidade para identificar a associação entre as variáveis por meio dos coeficientes de correlação de Pearson, com auxílio do software SPSS, sendo o mesmo procedimento realizado para os três produtos.

Com os resultados, foi possível observar que há correlação alta entre o orçamento promocional e o orçamento comercial para os produtos $\alpha(0,88), \beta$ $(0,96)$ e $\Omega(0,83)$ e correlação alta entre IGP e volume de vendas para os produtos $\alpha(0,81)$ e $\Omega(0,90)$. Em todos os casos, o p-valor foi inferior ao nível de significância adotado de 0,05 indicando que existe relação entre as variáveis observadas.

Neste caso, uma das variáveis com correlação alta poderia ser descartada do modelo de previsão pelo fato de não possuir alta correlação com a demanda ou possuir alta correlação entre si. Todavia, com base em Makridakis, Wheelwright e Hyndman (1998) as variáveis foram mantidas, pois não necessariamente as demais variáveis independentes forneceriam a melhor correlação com a venda. Desta forma, justificamse as variáveis independentes inseridas no modelo, sendo elas: preço, prazo, orçamento promocional, orçamento comercial, comissão, aumento salarial, número de vendedores, IGP, IAE e IVE.

Nesta etapa, foram coletadas 108 observações de quatro empresas participantes do jogo de empresas. Tais observações correspondem a 16 períodos de simulação. Os dados foram coletados em documentos com as decisões das empresas e em relatórios com os resultados fornecidos pelo simulador a cada período.

Com a aplicação da análise de regressão linear múltipla, observou-se que a significância observada nos três modelos foi inferior à significância esperada de 0,05 , demonstrando que os modelos de previsão são úteis para prever a demanda. Por este motivo, há evidências de que pelo menos uma variável de cada modelo está relacionada com a venda. A partir desta análise, foi possível prosseguir no desenvolvimento do modelo.

Foram estabelecidos os valores da constante $\left(\mathrm{b}_{0}\right)$ e dos coeficientes $\left(b_{i}\right.$ 's) de cada variável independente para os três tipos de produto, de acordo com as Funções 2,3 e 4 .

Função do modelo de regressão para o produto $\alpha$
Função do modelo de regressão para o produto $\beta$

$Y_{\beta}=-126193,877-4,126 X_{1}+61,953 X_{2}-0,005 X_{3}+0,002 X_{4}+$ $Y_{\beta}=-126193,877-4,126 X_{1}+61,953 X_{2}-0,005 X_{3}+0,002 X_{4}+$ (3)

$-216,685 X_{5}+415,682 X_{6}+374,794 X_{7}+829,687 X_{8}+407,752 X_{9}+104,601 X_{10}$

Função do modelo de regressão para o produto $\Omega$

$Y_{\Omega}=-141375,828-3,000 X_{1}+44,377 X_{2}+0,000 X_{3}+0,001 X_{4}+$ $Y_{\Omega}=-141375,828-3,000 X_{1}+44,377 X_{2}+0,000 X_{3}+0,001 X_{4}+$ (4)

$+127,829 X_{5}-20,902 X_{6}-1125,142 X_{7}+1073,539 X_{8}+279,233 X_{9}+119,496 X_{10}$

Nessa etapa, foi verificada a exatidão do modelo por meio da determinação do $\mathrm{R}^{2}$. Esse coeficiente determina o quanto o conjunto de variáveis independentes explica o modelo. O percentual de variabilidade da demanda descrito pelo modelo foi de $82 \%$ para o produto $\alpha, 75 \%$ para o produto $\beta$ e $89 \%$ para o produto $\Omega$.

Em geral, o $\mathrm{R}^{2}$ ajustado é inferior ao $\mathrm{R}^{2}$. Este deve ser utilizado para comparar modelos com diferentes quantidades de variáveis, porém não é o caso desse estudo.

$\mathrm{Na}$ última etapa, foram projetados os valores futuros da venda para os três tipos de produto para o período de um ano. $\mathrm{Na}$ seção seguinte serão apresentados 
os resultados das novas decisões fornecidos pelo simulador e serão comparados os resultados sem e com o uso do modelo de previsão dos três produtos.

\section{Análise Descritiva dos Resultados}

Após a definição das funções dos modelos, foi escolhida uma empresa para ser analisada. O critério de escolha da empresa foi a compra dos três produtos em todos os períodos analisados para evitar alterações nas decisões das indústrias. As decisões da empresa analisada que dizem respeito às variáveis independentes foram revisadas com base no modelo de previsão e as demais decisões da empresa e das empresas concorrentes foram mantidas.

Com isso, as rodadas do jogo de empresas para o período de um ano foram reprocessadas. Deve-se ressaltar que as alterações no conjunto de decisões de um produto foram testadas de forma individual e não os três produtos ao mesmo tempo.

Para aplicação do modelo, foi necessário estimar o cenário econômico a cada rodada. O IGP previsto foi calculado com base no IGP do período anterior mais 1,5 , tendo-se em vista uma tendência observada no comportamento do índice em períodos anteriores. A previsão do IAE e IVE foi fornecida pelo simulador no período anterior.

Buscou-se estimar a demanda para a empresa analisada com base na quantidade de produtos disponíveis a cada período, sendo considerada a compra realizada e o estoque.

O modelo foi utilizado para ajustar as decisões comerciais: preço, prazo, orçamento de marketing (promocional e comercial) e força de vendas (comissão). A seguir os ajustes serão detalhados, tendo-se em vista dois cenários: sem e com o uso do modelo de previsão.

\section{(a) Produto $\alpha$}

A Figura 2 apresenta o volume de vendas e o custo unitário nos dois cenários (com e sem o modelo de previsão de vendas). Pode-se observar que no primeiro período a empresa obteve o mesmo volume de vendas, pois o que se buscou foi a otimização dos recursos, uma vez que não era possível modificar a quantidade de produtos disponível para venda. No segundo período, com o apoio do modelo de previsão a empresa foi capaz de gerar a demanda necessária para vender o estoque disponível. No terceiro período, o volume de vendas com o uso do modelo de previsão foi inferior devido à ausência de estoque que já havia sido vendido no período anterior. No último período, com o uso do modelo de previsão foi possível gerar uma demanda muito superior.

Com o modelo de previsão foi possível perceber que a variável prazo e orçamento comercial tem maior influência na demanda. Desta forma, as decisões foram ajustadas de forma que os investimentos nas demais variáveis foram alocados para o orçamento comercial. Com isso, obteve-se um custo unitário inferior na maioria dos períodos resimulados. O custo unitário superior no terceiro período justificase pelo fato do número de unidades disponíveis em estoque sem o modelo de previsão ser muito superior, resultando na diluição do custo.

O saldo de caixa da empresa analisada apresentou um histórico negativo ao longo das últimas rodadas do jogo. Por este motivo, foi dado um foco maior à variável prazo sendo realizados alguns ajustes. Em alguns períodos simulados foi necessário dar ao cliente um prazo de recebimento maior para gerar maior demanda, ainda assim a empresa conseguiu se recuperar e atingir lucros líquidos superiores. 
Figura 2 . Volume de Vendas e Custo Unitário- Produto $\alpha$

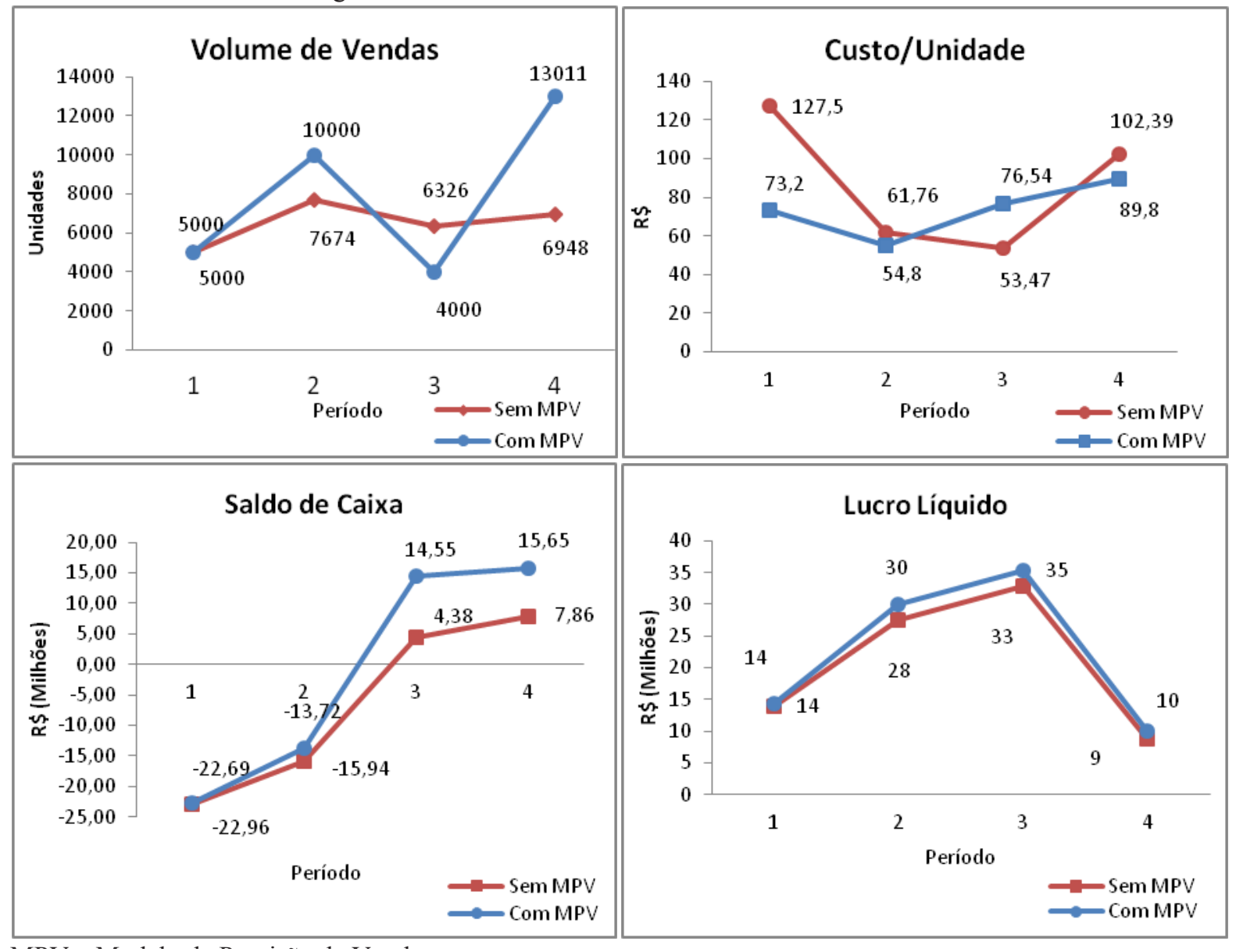

MPV = Modelo de Previsão de Vendas

Fonte: Elaboração própria.

\section{(b) Produto $\beta$}

Com o uso do modelo de previsão foi possível reduzir o estoque do produto $\square$ em todos os períodos. Para isso, os investimentos foram concentrados no orçamento promocional e no número de vendedores e aumento salarial. Da mesma forma, foi necessário aumentar o prazo de recebimento ao cliente para ampliar a venda, porém este não pôde ser estendido até 90 dias devido o saldo de caixa negativo acumulado.

Procurou-se manter o preço praticado sem o uso do modelo de previsão para manter o faturamento anterior e ajustar os investimentos realizados. O resultado pode ser observado na Figura 3 em que o custo unitário foi inferior, exceto na última rodada do jogo em que houve um pequeno aumento. Houve uma redução do saldo de caixa negativo de treze milhões de reais o que resultou em um aumento de cerca de seis milhões no lucro líquido acumulado. 
Figura 3. Volume de Vendas e Custo Unitário - Produto $\beta$

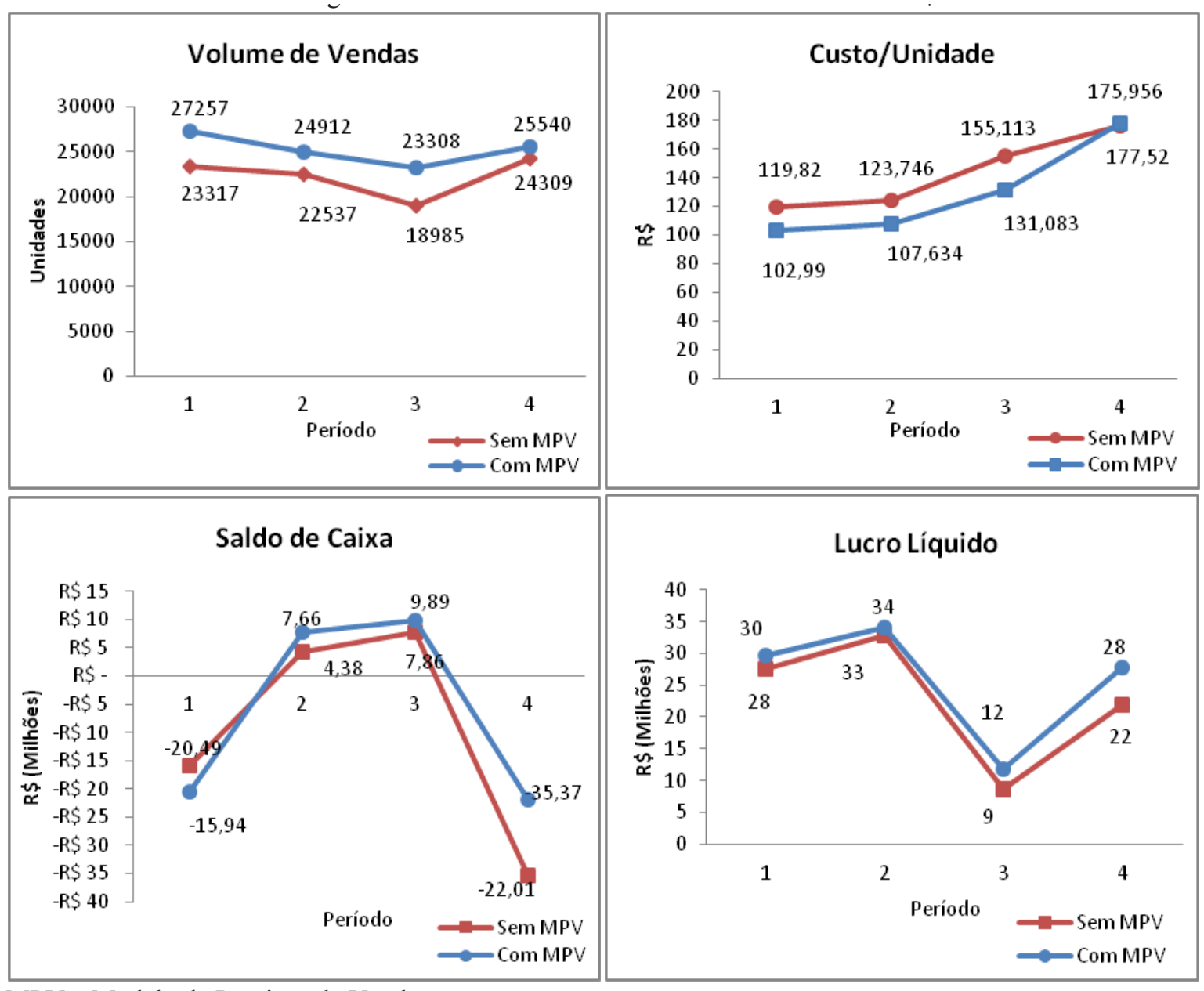

MPV $=$ Modelo de Previsão de Vendas.

Fonte: Elaboração própria.

\section{(c) Produto $\Omega$}

Com os ajustes nas decisões, foi possível aumentar o volume de vendas em todos os períodos analisados. Observou-se que embora o estoque estivesse muito elevado, a empresa comprou lotes altos nos demais períodos. É possível que a empresa tenha estabelecido uma parceria com o fornecedor e projetado a compra dos produtos em rodadas anteriores. Para eliminar o estoque seria necessário reduzir o preço de forma considerável, o que resultaria em prejuízos financeiros.

Para aumentar a demanda, foi necessário aumentar os investimentos na força de vendas (comissão), o que elevou de forma significativa os custos (Figura 4). O saldo de caixa acumulado obteve um aumento de noventa e dois milhões no último período analisado e o lucro líquido embora tenha acompanhado a evolução do anterior, resultou em um aumento de seiscentos mil. 
Figura 4 . Volume de Vendas e Custo Unitário - Produto $\Omega$

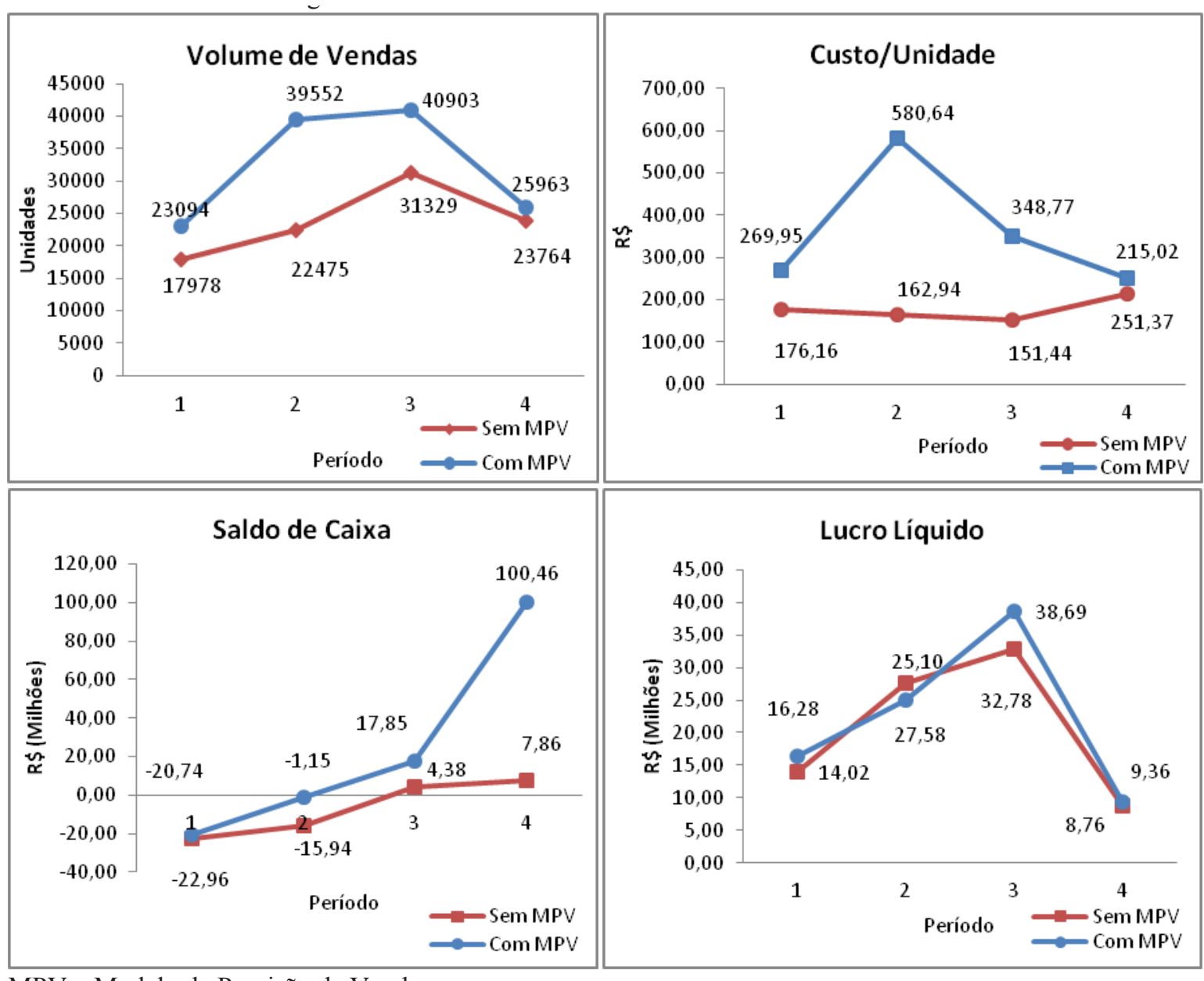

MPV = Modelo de Previsão de Vendas.

Fonte: Elaboração própria.

Para verificar a precisão do modelo desenvolvido, após as análises foram calculados os erros médios de previsão com o uso do modelo e comparado aos erros médios sem o uso do modelo de precisão para os três produtos.

Observou-se que o erro médio na previsão de vendas com o uso do modelo proposto foi muito inferior comparado ao erro no momento em que as empresas não usaram nenhum modelo de previsão de vendas. A diferença dos erros médios para os produtos $\alpha, \beta$ e $\Omega$ podem ser observados na Tabela 1 .

A previsão de vendas apresentou menor erro para os três produtos, com destaque para $\alpha$ e $\beta$. Embora o erro do produto $\Omega$ com o uso do modelo tenha sido muito superior comparado aos demais, o erro sem o modelo de previsão também foi muito superior. 
Tabela 1. Erro médio na previsão de vendas

\begin{tabular}{|c|cc|cc|cc|}
\hline Produto & \multicolumn{2}{|c|}{$\boldsymbol{\alpha}$} & \multicolumn{2}{c|}{$\beta$} & \multicolumn{2}{c|}{$\boldsymbol{\Omega}$} \\
Período & Sem & Com & Sem & Com & Sem & Com \\
& MPV $^{1}$ & MPV & MPV & MPV & MPV & MPV \\
\hline 1 & -100 & 0 & -29 & -3 & -151 & 39 \\
2 & -30 & 9 & -29 & 10 & -172 & 22 \\
3 & -1 & 0 & -107 & -18 & -135 & 46 \\
4 & -116 & -14 & -127 & -18 & -152 & 23 \\
\hline $\begin{array}{c}\text { Erro } \\
\text { Médio } \\
(\%)\end{array}$ & $\mathbf{6 1 , 7 5}$ & $\mathbf{5 , 7 5}$ & $-\mathbf{7 3}$ & $-\mathbf{7}$ & $-\mathbf{1 5 3}$ & $\mathbf{3 3}$ \\
\hline
\end{tabular}

${ }^{1}$ Modelo de Previsão de Vendas.

Fonte: Elaboração própria.

\section{Discussão dos Resultados}

Os resultados do estudo corroboraram com a literatura apresentada no referencial teórico. A tomada de decisão com base no modelo de previsão resultou em melhorias na empresa analisada em termos financeiros e operacionais, tendo o uso do modelo contribuído para uma boa gestão. Os resultados apresentados demonstraram que a análise de regressão pode ser utilizada para antecipar e prever a demanda futura.

Foi possível observar as variáveis independentes de maior influência no volume de vendas de cada produto, melhorando assim a alocação dos recursos na tomada de decisão. Para o produto alfa observou-se que a variável "prazo" tem forte influência, enquanto para o produto beta foram as variáveis "preço" e "número de vendedores" e para o produto ômega a variável "força de vendas" (comissão).

Com o uso do modelo, foi possível alinhar as necessidades do mercado (atender a demanda) à da empresa, sendo possível saldar os compromissos da empresa com o aumento do faturamento. Os resultados com uso do modelo de previsão de demanda em termos de saldo de caixa e custo por unidade foram superiores aos resultados da empresa sem uso do modelo, o que aponta o suporte dado pelo modelo na tomada de decisão.

Se a empresa analisada tivesse realizado o modelo de previsão de demanda durante o jogo de empresas, que é uma limitação deste estudo, seria possível realizar compras menores do produto ômega e aumentar o lucro líquido ao reduzir custos de estocagem ou realizar um planejamento mais elaborado de forma que maiores investimentos pudessem ser cobertos com o saldo de caixa positivo.

Confirmou-se o que havia sido declarado por Gerber et. al. (2013), que erros na previsão de demanda resultam em perdas financeiras, conforme foi possível observar no acúmulo negativo do saldo de caixa resultante do volume de vendas inferior ao esperado, sendo intensificado pela má alocação dos recursos.

A previsão tornou-se mais difícil devido a necessidade de se prever o cenário econômico, o que corroborou com a declaração de Melo e Alcântara (2011) quanto à ausência de informações e forte influência das variáveis exógenas. Embora as previsões da sazonalidade e da atividade econômica tenham sido fornecidas pelo simulador e a previsão do IGP tenha sido realizada com base em uma análise prévia do comportamento em períodos anteriores, as previsões estão sujeitos a erros.

Pode-se perceber que os ajustes nas decisões reduziram os custos unitários, porém não na mesma proporção como seria em uma economia de escala. Os custos associados à comercialização do produto ômega foram muito superiores quando comparados aos demais. Isso pode ser justificado pela necessidade de alto investimento na força de vendas (comissão) necessária para geração de demanda do produto.

Os ajustes nas decisões contribuíram para redução de custos, aumento das vendas e do lucro líquido da empresa analisada, o que corroborou com os autores que defendem o uso de um modelo de previsão.

\section{Considerações Finais}

O objetivo deste estudo foi desenvolver um modelo de previsão de vendas adequado ao jogo de empresas em estudo com uso de uma técnica quantitativa. Concluiu-se que é possível utilizar a análise de regressão linear para a construção de um modelo com $\mathrm{n}$ variáveis independentes para ser utilizado em um jogo de empresas. 
Pretendeu-se ainda identificar o grau de precisão do modelo e identificar alterações no desempenho da empresa analisada. Concluiu-se que o modelo foi capaz de prever a demanda obtendo um erro de previsão inferior ao erro no jogo de empresas sem o uso do modelo de previsão. O modelo contribuiu para um desempenho superior na empresa analisada em termos financeiros e operacionais.

Pode-se verificar o benefício decorrente do uso de um modelo estatístico para antecipar e prever a demanda, uma vez que o modelo agregou valor à empresa. Com isso, conclui-se que as empresas devem utilizar em seus processos de tomada de decisão modelos baseados em técnicas que sejam capazes de antecipar a demanda futura.

Os resultados da pesquisa mostraram que um modelo de previsão pode auxiliar as decisões estratégicas da empresa no curto e longo prazo e que as empresas devem dar a devida atenção à previsão de vendas.

Destaca-se, ainda, a contribuição desse estudo aos acadêmicos que tenham interesse em pesquisa e docência com uso da técnica jogo de empresas. O laboratório de gestão mostrou-se propício como ambiente de aprendizagem, tendo os participantes tido a oportunidade de vivenciar uma experiência significativa similar à realidade gerencial e colocar em debate teorias e conceitos aprendidos em sala de aula. Além de ser um ambiente para introduzir o estudante à pesquisa científica, coletar dados e testar conceitos e teorias.

Considera-se assim, o laboratório de gestão uma possibilidade para complementar a educação gerencial com uso de métodos ativos de aprendizagem, centrado no estudante, ao mesmo tempo que dá a ele a oportunidade de treinar e conhecer características da tomada de decisão, do ambiente empresarial e da prática dos gestores.

Os docentes e pesquisadores que tiverem interesse no tema podem usufruir desse estudo para obter reflexões acerca de como ocorre a dinâmica do jogo de empresas e seus benefícios, entre eles, a possibilidade de se integrar conteúdos na formação de gestores, obter uma visão sistêmica das organizações, associar teoria e prática e educar de forma lúdica.

A principal limitação do estudo resultou no fato de não ter sido realizado um teste durante o jogo de empresas. Pelo fato das decisões terem sido ajustadas e reprocessadas no simulador após o processo de tomada de decisão das equipes, além de terem sido resimulados apenas quatro períodos. Outra limitação refere-se à imprecisão do cenário econômico, o que comprometeu as previsões resultantes do modelo.

O baixo número de observações utilizado dificultou a precisão do modelo, além do número elevado de variáveis envolvidas que tornou o modelo mais complexo pela necessidade de se prever também as variáveis independentes. A análise do desempenho se limitou ao volume de vendas e ao resultado operacional e financeiro, não sendo considerada a capacidade do fornecedor e possíveis parcerias pré-estabelecidas.

E, por fim, outra limitação deve-se ao fato de algumas variáveis terem apresentado não linearidade no teste de normalidade realizado.

Como proposições para novos estudos, sugerese a combinação do modelo utilizado com técnicas qualitativas para aumentar a precisão. O julgamento de especialistas poderia auxiliar na previsão do cenário econômico e de possíveis acontecimentos ao longo do jogo, conforme discutido no referencial teórico desse estudo.

Poderiam ser utilizadas técnicas de tratamento estatístico não linear para o desenvolvimento do modelo e o uso do método Best Subset para seleção das variáveis independentes.

\section{Referências}

BEN-ZVI, T. The efficacy of business simulation games in creating Decision Support Systems: An experimental investigation. Decision Support Systems, 49, p. 61-69, 2010.

DIEGUEZ-BARREIRO, J. H.; GONZÁLEZBENITO, J.; GALENDE, J. The use of management games in the Management research agenda.

Developments in Business Simulation and Experiential Learning, v. 38, 2011.

GERBER, J. Z.; MIRANDA, R. G.; BORNIA, A. C.; FREIRES, F. G. M. Organização de Referenciais 
Teóricos Sobre Diagnóstico Para a Previsão de Demanda. Gestão Organizacional - Recife/PE Brasil, v. 11, n. 1, p. 160 - 185 Jan./Abr. 2013.

GOLD, S.; PRAY, T. Inside the black box: an analysis of underlying demand functions in contemporary business simulations. Proceedings of the Association for Business Simulation and Experiential Learning Conference, Phoenix, AZ, USA, 9, 1982.

GOLD, S.; PRAY, T. Modeling non-price factors in the demand functions of computerized business simulations. Proceedings of the Association for Business Simulation and Experiential Learning Conference, Stillwater, OK, USA, 11, 1984.

GOODWIN, P. Integrating management judgment with statistical methods toimproveshorttermforecasts. Omega - International Journal of Management Science, 30, p. 127-135, 2002.

GOOSEN, K. A Generalized Algorithm For Designing And Developing Business Simulations. Developments In Business Simulation and Experiential Exercises, v. 8, 1981.

HONAISER, E. H. R.; SAUAIA, A. C. A. Desenvolvimento e Aplicação de um Modelo para Previsão de Demanda em Jogos de Empresas. RACEletrônica, Curitiba, v. 2, n. 3, art. 7, p. 470-485, Set./Dez. 2008.

LAMBERT, N. E.; LAMBERT, D. R. Advertising Response In The Gold And Pray Algorithm: A Critical Assessment. Developments in Business Simulation \& Experiential Exercises, v. 15, 1988.

LIN, T. Y. Estudo de modelos de previsão de demanda. Núcleo de Pesquisas e Publicações da FGV-EAESP. v. 3, n. 1, p. 25-29, 2000.

MAKRIDAKIS, S.; WHEELWRIGHT, S.; HYNDMAN, R. J. Forecasting Methods and Applications. John Wiley \& Sons. 3a Edição. New York, 1988.

MELO, D. C.; ALCANTARA, R. L. C. A gestão da demanda em cadeias de suprimentos: uma abordagem além da previsão de vendas. Gestão e Produção, São Carlos, v. 18, n. 4, p. 809-824, 2011.
OLIVEIRA, M. A.; SAUAIA, A. C. A.; MOT'TA, G. S.; GARCIA, P. A. A. Integração entre Educação Gerencial e Pesquisa em Administração: um estudo sobre o Desenvolvimento de um Laboratório de Gestão. Iberoamerican Academy of Management (IAM) 7th International Conference. December 5-7, Lima, Peru, 2011.

PORTER, A.; ROPER, T.; NASAN, T.; ROSSINI, F. Forecasting and management technology. New York: John Willey and Sons Press, 1991.

PRAY, T.; GOLD, S. Inside the Black Box - An Analysis of Underlying Demand Functions in Contemporary Business Simulations. Developments In Business Simulation and Experiential Exercises, v. 9, p. 110-115, 1982.

PROTO, L. O. Z.; MESQUITA, M. A. Previsão de demanda para planejamento da capacidade de empresa do setor cimenteiro. XXIII Encontro Nacional de Engenharia de Produção - Ouro Preto, MG, Brasil, 21 a 24 de out de 2003.

ROSAS, A. R. Criação de um Simulador Educacional para Empreendedores: simulando novos negócios B2B de base tecnológica. Tese. (Doutorado). Universidade de São Paulo. São Paulo, SP, Brasil, 2009.

SAUAIA, A. C. A. Satisfação e aprendizagem em jogos de empresas: contribuições para a educação gerencial. Tese. (Doutorado). Universidade de São Paulo, Faculdade de Economia, Administração e Contabilidade, São Paulo, SP, Brasil, 1995.

\section{SHADOW MANAGER. Manual do Simulador}

Shadow Manager. Simulador Pedagógico de Gestão Empresarial. Versão 5.7.

SILVA, S. S.; OLIVEIRA, M. A.; MOTTA, G. S. Jogos de Empresas e Método do Caso: Contribuições ao Processo de Ensino e Aprendizagem em Administração. Revista Administração: Ensino e Pesquisa-RAEP, v. 14, n. 4, p. 667-705, 2013.

SYNTETOS, A. A.; NIKOLOPOULOS, K.; BOYLAN, J. E.; FILDES, R.; GOODWIN, P. The effects of integrating management judgement into intermittent demand forecasts. Int. J. Production Economics, 118, p. 72-81, 2009. 
THAVIKULWAT, P. Simulating demand in an independent across-firms management game.

Proceedings of the Association for Business

Simulation and Experiential Learning

Conference, Stillwater, OK, USA, 15, 1988.

WEBBY, R.; O'CONNOR, M. Judgement and

Statistical Time Series Forecasting: a Review of the

Literature. International Journal of Forecasting, v.

12, p. 91-118, 1996.

WERNER, L.; RIBEIRO, J. L. D. Modelo composto

para prever demanda através da integração de

previsões. Produção, v. 16, n. 3, p. 493-509, Set./

Dez. 2006. 